Pacific Journal of Mathematics

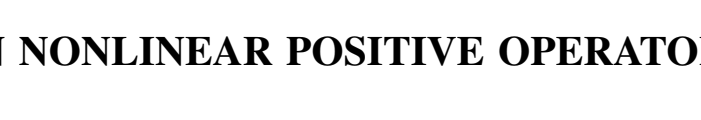




\section{ON NONLINEAR POSITIVE OPERATORS}

\section{H. H. SchaEFER}

Introduction. The purpose of the present paper is to apply the well known Schauder fixed point theorem, in its general from due to Tychonov [8], to the situation of nonlinear (or rather, not necessarily linear) maps defined on (or on a subset of) the "positive" cone in a partially ordered locally convex linear space. Throughout this paper, no use is made of possible linear properties of the maps under consideration. As far as the author is informed, there is little history to the study of such mappings; the only work done seems to be contained in papers by Krein-Rutman [2], Rothe [9] and Morgenstern [3]. In [2], the Schauder theorem is largely applied to linear maps (where it can be avoided) and a few nonlinear cases ${ }^{1}$. In [4], the author paid attention mainly to the case of linear compact maps in general locally convex spaces. At the end of that paper, with a somewhat sketchy proof, a general nonlinear theorem ${ }^{2}$ is stated which however seems to need some improvement.

In this paper, the essential proposition resulting from the fixed point theorem is stated in the form of three different theorems to throw some light on potential ways of argument. While Th. 1, depending on a special convexity argument, is of a different character, Th. 2 is almost a special case of Th. 3. But as Banach spaces with normal order cones (with which Th. 2 is concerned) seem to be the most important ones in nonlinear analysis, it might be useful to have the theorem stated separately, a much simpler proof than that of Th. 3 going with it. Applications have been selected so as to furnish a non-trivial example to each of the three theorems, the one to Th. 1 showing that it is not always fruitful to restrict attention to normed topologies. It is understood that each example constitutes a new result in its respective field.

Preliminary material. In the present section, we are going to collect some theorems and definitions on which argumentation will be primarily based in the sections to follow. The main tool will be the

Fixed Point Theorem (Tychonov). Let $E$ be a locally convex linear space, $M$ a convex compact subset of $E$. If $T$ is a continuous map on $M$ into $M$, then $T$ has a fixed point $x_{0} \in M$.

Received September 29, 1958, and in revised form February 11, 1959.

1 Also, considerations are restricted to Banach spaces.

2 Satz 3.1. This is restated and proved in this paper as Th. 3. The additional assumption to be made in [4] may be any one of hypotheses $\alpha, \beta$ stated with Th. 3 of the present paper. 
For the proof, see [8]. To make this theorem more easily applicable to mappings that carry sets not necessarily compact into compact ones, we state the following slightly more general

Fixed Point Theorem (2nd form). Let $M$ be a complete convex set in $E$. If $T$ is continuous on $M$ into $M$ such that $T(M)$ is relatively compact, then $T$ has a fixed point $x_{0} \in M$.

Proof. Let $M_{1}$ be the closed convex hull of $T(M)$. As $M$ is complete, $M_{1}$ is compact (Bourbaki [1], p. 81) and since obviously $T\left(M_{1}\right) \subset M_{1}$, Tychonov's theorem yields the desired result.

Let $E$ be a linear space over the real scalar field. A partial ordering of $E$ is a binary relation " $<$ " such that

$$
x<x \text { for all } x \in E .
$$

$\{x<y \& y<z\} \Rightarrow x<z$.

3

$$
\{x<y \& y<x\} \Rightarrow x=y \text {. }
$$

Such an ordering is said to be compatible with the linear structure of $E$ if in addition

$$
\begin{aligned}
& \{x>0 \& \lambda \geqq 0\} \Rightarrow \lambda x>0 . \\
& x>y \Rightarrow x+z>y+z
\end{aligned}
$$$$
\text { for all } z \in E \text {. }
$$

The set of all $x \in E$ such that $x>0$ is a convex cone $C$ which contains its vertex 0 , and which is proper (i.e. $C \cap-C=\{0\}$ ). $C$ will be referred to as the positive cone with respect to a given partial ordering of $E^{3}$ ). Conversely, each cone in $E$ with the listed properties defines a partial ordering satisfying axioms 1 through $5, x<y$ meaning $y-x \in C$.

Let $E$ be a linear space, partially ordered by some such cone $C$. If $T$ is a mapping defined on a subset of $C$, we will say $T$ is positive whenever the range of $T$ is in $C$. If $E$ is, moreover, a topological space, $T$ will be called strictly positive if $T\left(x_{n}\right) \rightarrow 0$ implies $x_{n} \rightarrow 0$ for any sequence $\left\{x_{n}\right\}$ in the domain of $T$.

\section{Examples.}

1. Let $E$ be Hilbert space $L_{2}(0,1)$ in its natural order, i.e. the positive cone $C$ consisting of all elements $f: f(t) \geqq 0, t \in[0,1]$. The positive mapping, defined on all of $E$,

$$
T(f)=\int_{0}^{t} f^{2}(\tau) d \tau
$$

${ }^{3}$ In this paper, all orderings are understood to be compatible with the linear structure of the space involved. Also, we exclude the trivial case $C=\{0\}$. 
is not strictly positive. Take $f_{n}=\sqrt{n t^{n-1}}$, then $\left\|f_{n}\right\|=1$. Now as

$$
T\left(f_{n}\right)=t^{n},\left\|T\left(f_{n}\right)\right\|=\frac{1}{\sqrt{2 n+1}} \rightarrow 0
$$

2. Let $E$ be the $B$-space of continuous functions on the unit interval, with its natural partial order. Let $K(t, \tau)$ be $\geqq 0$ (but $\not \equiv 0$ ) and continuous on the unit square. If $P(z)$ is a polynomial with non-negative coefficients,

$$
T(f)=\int_{0}^{1} K(t, \tau) P[f(\tau)] d \tau
$$

is strictly positive if and only if the constant term in $P$ is $>0$.

3. Denote by $\left\{E_{\alpha}\right\}$ a collection of topological linear spaces, each $E_{\alpha}$ being partially ordered by some positive cone $C_{\alpha}$. Then the product space $E=\prod_{\alpha} E_{\alpha}$ is ordered by $C=\prod_{\alpha} C_{\alpha}$. Let $A_{\alpha}$ be a positive map on $E$ into $E_{\alpha}$, and consider the map

$$
A(x)=\left(\cdots, A_{\alpha}(x), \cdots\right)
$$

on $E$ into $E$. Then $A$ is strictly positive on $C$ if and only if to each $\alpha$, there is a $\beta(\alpha)$ such that $A_{\beta}(x) \rightarrow 0$ (in $E_{\beta}$ ) implies $x_{\alpha} \rightarrow 0$ (in $E_{\alpha}$ ). In particular, if $A_{\alpha}(x)=A_{\alpha}\left(x_{\alpha}\right)$, then $A$ is strictly positive if and only if each $A_{\alpha}$ is.

I. Morgenstern's theorem. If $E$ is the Banach space $L_{1}$, partially ordered by the positive cone $C=\{f: f(t) \geqq 0\}$, it turns out that the intersection of $C$ with the unit sphere $S=\{f:\|f\|=1\}$ is convex. This is true for any abstract $L$-space or, more generally, for any normed space in which the norm is additive on $C$. To this situation Morgenstern [3] applied Schauder's fixed point theorem. He obtained the following

THEOREM (Morgenstern) ${ }^{4}$. Let E be a Banach space, partially ordered by a positive cone $C$ which is closed and on which the norm is additive. Then if $T$ is continuous and strictly positive on $C \cap\left\{\|x\|=c_{j}\right.$, $c>0$, mapping this set into a compact one, there is some $\lambda>0$ and $x \in C$ such that $\lambda x=T(x),\|x\|=c$.

The proof is readily obtained by applying the fixed point theorem (2nd form) to the map $c T(x) /\|T(x)\|$ on the set $C \cap\{\|x\|=c\}$. However, it may be so arranged as to yield a much more general proposition.

${ }^{4}$ The theorem is stated in our terminology and a slightly more general form. 
THeOREM 1. Let $E$ be a locally convex space, partially ordered by a complete cone $C$, and let $T$ be a continuous, strictly positive transformation on $C$, mapping bounded sets into compact ones. Assume $H: f(x)=1$ is a closed hyperplane meeting $C$ in a nonvoid, bounded set. Then to each $c>0$, there is an $x \in C$ and $\lambda>0$ with

$$
\lambda x=T(x), \quad f(x)=c .
$$

Proof. It follows from our assumptions that the continuous linear form $f(x)$ is $>0$ at every non-zero point of $C$. For assume there is an $x_{0} \in C, x_{0} \neq 0$, with $f\left(x_{0}\right)=0$. Then if $y_{0} \in H \cap C$, we would have $f\left(y_{0}+\mu x_{0}\right)=1$ for all $\mu \geqq 0$ which contradicts the hypothesis that $H \cap C$ be bounded. It is now also clear that $f$ cannot be $<0$ on $C$. Applying the fixed point theorem (2nd form) to the map $c T(x) / f[T(x)]$ on the set $H \cap C$, we get the desired result letting $\lambda=f[T(x)] . c^{-1}$.

REMARK. We should point out the relation between Morgenstern's theorem and Th. 1. If, under the assumptions of the former, the norm coincides on $C$ with a continuous linear form, then Morgenstern's theorem is a corollary of Th. 1. (This is the case in $L_{1}$, e.g.). Assume then, still under the assumptions of Morgenstern's theorem, that there is no such linear form. Now $C \cap\{\|x\|=c\}$ is convex $(c>0)$, so there is a closed hyperplane $H$ separating this set from a convex open neighborhood of 0 . Obviously $H \cap C$ is bounded and Th. 1 can be applied provided $T$ is compact, continuous and strictly positive on $C$.

II. Banach spaces with normal positive cones. We will now extend Morgenstern's theorem to ordered Banach spaces in which the norm is not necessarily additive on the positive cone $C$. This assumption will be replaced by the weaker hypothesis that $C$ is normal. A convex cone of vertex 0 in a normed space $E$ is normal [5] if the topology of $E$ is generated by a norm which is monotone (with respect to the order induced in $E$ by $C$ ) on $C$. In terms of the given norm on $E, x \rightarrow\|x\|$, this amounts to saying there is a constant $\gamma>0$ such that

$$
\|x+y\| \geqq \gamma\|y\| \quad \text { for all } x \in C, y \in C .
$$

It can easily be checked that for all classical Banach spaces, the positive cones pertaining to their natural partial orders are normal ([5], p. 130).

THEOREM 2. Let $E$ be a normed space, partially ordered by a complete normal cone $C$. Let $T$ be a strictly positive transformation, which is continuous and maps bounded subsets of $C$ into compact ones. Then to each $c>0$, there is $x \in C$ and $\lambda>0$ with

$$
\lambda x=T(x), \quad\|x\|=c .
$$


Proof. Consider the mapping

$$
x \rightarrow S_{c}(x)=T(x)+|c-\|x\|| y
$$

for fixed $0 \neq y \in C$ and $c>0$. This is a continuous map carrying bounded subsets of $C$ into compact ones. $T$ being strictly positive, we have $\inf \left\{\|T(x)\|: x \in C \&\|x\| \geqq \frac{1}{2} c\right\}=\varepsilon>0$. Hence, $C$ being a normal cone, we obtain

$\inf _{x \in O}\left\|S_{c}(x)\right\| \geqq \gamma \cdot \inf _{x \in o} \sup (\|T(x)\|, \mid c-\|x\|\|\| y \|) \geqq \gamma \sup \left(\varepsilon, \frac{1}{2} c\|y\|\right)>0$.

Thus $x \rightarrow R_{c}(x)=c S_{c}(x)\left\|S_{c}(x)\right\|^{-1}$ maps $C \cap\{\|x\| \leqq c\}$ into a compact subset. So by the fixed point theorem (2nd form) there is an $x$ in this subset with $x=R_{c}(x)$. Clearly $\|x\|=c$, and letting $\lambda=c^{-1}\left\|S_{c}(x)\right\|$ we have $\lambda x=T(x)$. Since $c>0$ is arbitrary, the proof is complete.

III. A third theorem. The theorem presented in this section weakens the assumption in Th. 2 that $E$ be normed and removes the hypothesis that $C$ be a normal cone. Instead, we require either one of conditions $\alpha, \beta$ of hypothesis $H$ (s. below) to hold. As the conclusion is only established for some continuous semi-norm $x \rightarrow p(x)$ on $E$ (which, however, may be assumed to generate the topology of $E$ if $E$ is normed), Th. 3 is not a generalization of Th. 1 or 2 . We start out with a

Lemma. If $E$ is a locally convex space, $C$ a closed proper convex cone in $E$ of vertex 0 , then there exists a continuous linear form on $E$, non-negative on $C$ and $>0$ at a given non-zero element of $C$.

Proof. Let $0 \neq y \in C$. Since $C$ is proper and closed, there is a convex open neighborhood $U$ of $-y$ such that $C$ and $\bigcup_{\lambda>0} \lambda U$ do not intersect. Hence there is a closed hyperplane $H$ separating $C$ and $\mathbf{U}_{\lambda>0} \lambda U$. Obviously $H$ contains 0 , so has an equation $f(x)=0$. After a potential change of sign, $f$ will meet the requirement.

Now let $E$ be any locally convex space, partially ordered by a complete positive cone. A mapping $T$, defined on a neighborhood of 0 in $C$ into $C$, will be called of type " $P$ " if it satisfies:

1. $T$ is continuous and strictly positive.

2. There is a neighborhood $U$ of 0 such that the image under $T$ of $U \cap C$ is relatively compact.

Consider

Hypothesis H. We will say that hypothesis $\mathrm{H}$ is satisfied if one of 
the two following statements is true:

$\alpha$. To each compact subset of $C$, there exists a continuous seminorm which is $>0$ at each non-zero point of that set.

$\beta$. $T$ is positive-homogeneous of some degree $\sigma>0$, i.e. $T(\lambda x)=$ $\lambda^{\sigma} T(x)$ for $x \in C$ and $\lambda>0$.

For instance, condition $\alpha$ is automatically fulfilled if there exists a continuous norm on $E$ (or even on $C)^{5}$. Condition $\beta$ is of course satisfied if $T$ is a linear map.

Theorem 3. Assume hypothesis $\mathrm{H}$ holds and $T$ is a mapping of type "P". Then there exists a continuous semi-norm $p$ such that for each $0<c \leqq 1$, there are an $x \in C$ and $\lambda>0$ satisfying

$$
\lambda x=T(x), \quad p(x)=c .
$$

Proof. Let $U=\left\{x: q_{1}(x) \leqq 1\right\}$ be a closed neighborhood of 0 such that $T(U \cap C)$ is relatively compact. Second, let $q_{2}$ be selected, according to which one of conditions $\alpha, \beta$ in $\mathrm{H}$ is satisfied, as follows:

Case $\alpha$. Let $q_{2}$ be a continuous semi-norm strictly positive on $T(U \cap C)$.

Case $\beta$. Let $q_{2}=q_{1}$.

Third, by the lemma, we may choose an $y \in C$ and a continuous linear form $f$ such that $f \geqq 0$ on $C$ while $f(y)>0$. We may further suppose that $\sup \left\{q_{1}(y), q_{2}(y), f(y)\right\}=1$.

Put $p=\sup \left\{q_{1}, q_{2},|f|\right\}$ and consider the set $U_{1}=\{x \in C: p(x) \leqq c\}$, $c$ being any fixed real number between 0 and 1 (1 included). For any positive integer $n$, form the mappings

$$
T_{n}(x)=T(x)+|1-p(T x)| \cdot n^{-1} y
$$

and $S_{n}(x)=c T_{n}(x) / p\left[T_{n}(x)\right]$. Obviously, $S_{n}$ is a transformation of type " $P$ ", mapping $U_{1}$ into itself, provided the denominator $p\left[T_{n}(x)\right]$ has a positive lower bound. To show that this is true, consider first all $x \in U_{1}$ such that $p(T(x)) \leqq \frac{3}{4}$. Then

$$
p\left(T_{n}(x)\right) \geqq f\left[T_{n}(x)\right] \geqq \frac{1}{4 n} f(y) \neq 0 .
$$

For the remaining elements $x \in U_{1}$ we have $p(T(x))>\frac{3}{4}$, hence

$$
p\left(T_{n}(x)\right) \geqq p(T(x))-|1-p(T(x))| n^{-1} p(y)>\frac{3}{4}-\frac{1}{4}=\frac{1}{2} .
$$

${ }^{5}$ Condition $\alpha$ can be weakened so as to require the existence and continuity of the semi-norms involved only on $C$. 
Applying the fixed point theorem (2nd form) to $S_{n}$, we are sure there is an $x_{n} \in U_{1}$ satisfying $x_{n}=S_{n}\left(x_{n}\right), p\left(x_{n}\right)=c$. Letting $\lambda_{n}=p\left(T_{n}\left(x_{n}\right)\right) \cdot c^{-1}$, by definition of $S_{n}$ we obtain

$$
\lambda_{n} x_{n}=T\left(x_{n}\right)+\left|1-p\left(T\left(x_{n}\right)\right)\right| n^{-1} y .
$$

$\left\{T\left(x_{n}\right)\right.$; being relatively compact, it follows that $\left\{\lambda_{n}\right\}$ is a bounded sequence. Assume, for the moment, that $\left\{\lambda_{n}\right\}$ has a positive lower bound. Then as the right-hand side of our last equation is relatively compact, so is $\left\{x_{n}\right\}$. (Here we may remark that for a convergent subsequence of $\left\{x_{n}\right\}$, the corresponding subsequence of $\lambda$ 's converges automatically to some $\lambda>0$.) Hence for each limiting point of a subsequence of $\left\{\lambda_{n} x_{n}\right\}$, such that $\lambda_{n_{k}} \rightarrow \lambda, \lambda x=T(x)$ and, by continuity, $p(x)=c$.

All that remains to prove is that $\lambda_{n}>\eta>0$ for all $n$. Suppose there were a subsequence $\left\{\lambda_{k}\right\}$ tending to zero. From this it would follow that $p\left(T\left(x_{k}\right)\right) \rightarrow 0$ which, by definition of $p$, in turn would imply $q_{2}\left(T\left(x_{k}\right)\right) \rightarrow 0$. On the other hand, $T$ being strictly positive, 0 is no limiting point to the sequence $T\left(x_{n}\right)$ because of $p\left(x_{n}\right)=c$. Thus if $\alpha$ of $\mathrm{H}$ is satisfied, we arrive at a contradiction. Now assume $\mathrm{H}$ holds by virtue of condition $\beta$. Letting $z_{n}=\lambda_{n} x_{n}$, $\left\{z_{n}\right\}$ has a limiting point $z$, say. Because $T$ is strictly positive, we must have $z \neq 0$. Multiplying equation $\left.{ }^{*}\right)$ by $\lambda_{n}^{\sigma}$, we get

$$
\lambda_{n}^{\sigma} z_{n}=T\left(z_{n}\right)+o\left(\frac{1}{n}\right) y
$$

Now if there were any subsequence $\left\{\lambda_{k}\right\}$ of $\left\{\lambda_{n}\right\}$ such that $\lambda_{k} \rightarrow 0$, we would obtain (as $\lambda_{k}^{\sigma} \rightarrow 0$ ) $T(z)=0$ for some $z \in C, z \neq 0$. This again contradicts the hypothesis that $T$ be strictly positive, and the proof is complete.

Remark. Hypothesis $H$ was needed to prove that $\left\{\lambda_{n}\right\}$ does not have 0 as a limiting point. The proof of Satz 3.1 in [4] is essentially the same as the one presented here, but is incorrect at the point where it says " $\lambda_{0}>0$ " (l.c., p. 329 , line 3 f.b.).

Applications. The remainder of this paper is concerned with a number of applications to the preceding theorems.

1. Consider the linear space $\omega$ of all real sequences $x=\left(x_{1}, x_{2}, \cdots\right)$, partially ordered by the positive cone $C=\left\{x: x_{i} \geqq 0, i=1,2, \cdots\right\}$. In the product topology (i.e. considering $\omega$ the product of countably many real lines) $\omega$ is locally convex. Let $r>0$ be a fixed integer and let $k=\left(k_{1}, k_{2}, \cdots\right)$ denote any sequence of non-negative integers such that 
$\sum_{i=1}^{\infty} k_{i}=r .^{6}$ Since the set $\{k\}$ of all such $k$ is countable, we may arrange it into a sequence; hence consider $\{k\}$ as ordered by the natural order of subscripts.

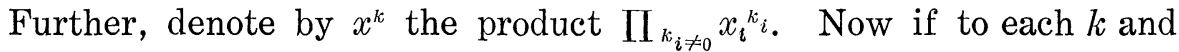
each positive integer $i$ there corresponds a real number $a_{i k} \geqq 0$ such that

$$
\sum_{(k)} a_{i k}<C
$$

where $C$ is independent of $i$, the equations

$$
y_{i}=\sum_{(k)} a_{i k} x^{k}
$$

define a mapping $y=A(x)$ on the subspace of all bounded sequences into itself such that each $y_{i}$ is a homogeneous form of degree $r$ in the variables $x_{1}, x_{2}, \cdots$ (If $r=1$, then $A$ defines a bounded linear map on the $B$-space $(m))$.

Consider the properties

$\alpha$. There are $n$ rows in $A$ (the first $n$ rows, say) such that

$$
\sum_{i=1}^{n} a_{i k} \geqq a_{j k} \quad \text { for all } k \text { and all } j>n .
$$

B. $\sum_{i=1}^{n} y_{i} \rightarrow 0$ implies $\sum_{i=1}^{n} x_{i} \rightarrow 0^{7}$.

We prove the following theorem:

If a mapping $A$ of the above mentioned type satisfies $\alpha$ and $\beta$, there are $a \lambda>0$ and an $x>0$ for which

$$
\lambda x=A(x) .
$$

REMARK. If $r=1$, then the point spectrum of the bounded map $A$ on $(m)$ contains a positive real number.

Proof. Consider in $\omega$ the cone $C_{1}=C \cap\left\{x: \sum_{i=1}^{n} x_{i} \geqq x_{j}, j>n\right\}$. Owing to $\alpha, A(x)$ is defined on the cone $C_{1}$ into itself. Since $\omega$ is complete and $C_{1}$ closed, $C_{1}$ is a complete cone in $\omega$. Next we show that $A$, which is in general not defined but on a dense subset of $\omega$, is continuous on $C_{1}$. Let $x_{n} \rightarrow x$ in $C_{1}$. It follows from the definition of $C_{1}$ that all coordinates of all the $x_{n}$ are uniformly bounded, say by some

6 A more general theorem results if we admit all $k$ such that $\sum_{i=1}^{\infty} k_{i} \leqq r$.

$7 \mathrm{Cf}$. Example 3 in the preliminary section. 
constant $M \geqq 1$. Given $\varepsilon>0$ and any fixed subscript $i$, we can find a $k_{0}$ such that

$$
\sum_{k>k_{0}} a_{i k}<\frac{\varepsilon}{3 M^{r}}
$$

Then if $y_{n}=A\left(x_{n}\right)$ and $y=A(x)$, we obtain

$$
\left|\left(y_{n}-y\right)_{i}\right| \leqq \sum_{k \leqq k_{0}}\left|a_{i k} x_{n}^{k}-a_{i k} x^{k}\right|+\sum_{k>k_{0}} a_{i k} x_{n}^{k}+\sum_{k>k_{0}} a_{i k} x^{k}
$$

The last two righthand terms are, by the choice of $k_{0}$, each less than $\frac{1}{3} \varepsilon$. The first righthand term will be less than $\frac{1}{3} \varepsilon$ for $n>n_{0}$ if $n_{0}$ is large enough, since there are only finitely many coordinates of both $x_{n}$ and $x$ involved. Thus we have $\left|\left(y_{n}-y\right)_{i}\right|<\varepsilon$ if $n>n_{0}$ and continuity is established.

Now $f(x)=\sum_{i=1}^{n} x_{i}$ is a continuous linear form on $\omega$. The intersection of the hyperplane $f(x)=1$ with $C_{1}$ is certainly bounded as $(0 \leqq) x_{i} \leqq$ $1, i=1,2, \cdots$, in that intersection. Moreover, a set $\{x\}$ is bounded in $\omega$ if and only if $\left|x_{i}\right|<M_{i}$ uniformly on $\{x\}$. If $M_{\imath}$ can be chosen independently of $i$, then the set is relatively compact by the well known Tychonov theorem. Thus on $C_{1}$ closed bounded sets coincide with compact sets, and $A$ transforms bounded sets into compact ones on $C_{1}$.

By hypothesis $\beta, A$ is strictly positive on $C_{1}$. (Conditions more explicit than $\beta$ may be obtained easily by applying the reasoning of Example 3, preliminary section.) Hence $A$ meets all the requirements of Th. 1 and the proof is complete.

2. In a recent paper [7], Schmeidler proved the existence of an eigenvalue to the homogeneous algebraic integral equation of order $n$

$$
\mu^{n} y^{n}(s)-\sum_{\beta=0}^{n-1} \mu^{\beta} y^{\beta}(s) a_{\beta}(s, y)=0, \quad(0 \leqq s \leqq 1)
$$

where $n$ is an odd integer $>0$ and

$$
a_{\beta}(s, y)=\int_{0}^{1} \cdots \int_{0}^{1} K_{\beta}\left(s, t_{1}, \cdots, t_{\nu}\right) y^{3+1}\left(t_{1}\right) \cdots y^{3+1}\left(t_{\nu}\right) d t_{1} \cdots d t_{\nu}
$$

are homogeneous integral forms with continuous kernels $K_{\beta}\left(s_{1} t_{1} \cdots t\right.$.) such that $(\beta+1)(\nu+1)=n+1$ and the $K$ 's are symmetric with respect to all their arguments. Schmeidler shows $(*)$ to be the natural generalization of a linear Fredholm equation with continuous symmetric kernel. In an earlier paper [6], a theorem was stated by Schmeidler that generalizes the well known Jentzsch theorem on linear Fredholm integral equations with positive kernel. The proof of that theorem of 
Schmeidler's, however, appears to be incorrect ${ }^{8}$. We are going to show that the theorem yet is correct and holds under weaker conditions than the ones stated in [6]. Let us call $\left(^{*}\right)$ an algebraic integral equation with non-negative coefficients if $n$ is any positive integer and

$$
a_{\beta}(s, y)=\sum_{\alpha_{1}+\cdots+\alpha_{\nu}=n-\beta} \int_{0}^{1} \cdots \int_{0}^{1} K_{\beta \alpha_{1}} \cdots \alpha_{\nu}\left(s, t_{1}, \cdots, t_{\nu}\right) y\left(t_{1}\right)^{\alpha_{1}} \cdots y\left(t_{\nu}\right)^{x_{\nu}} d t_{1} \cdots d t_{\nu}
$$

are homogeneous integral forms of order $n-\beta$ with continuous kernels $K_{\beta \alpha_{1} \cdots \alpha_{\nu}}\left(s, t_{1}, \cdots, t_{\nu}\right) \geqq 0$. We will prove this theorem:

If some $\alpha_{\beta}$ contains a term $\int K\left(s, t_{1}, \cdots, t_{\nu}\right) y\left(t_{1}\right)^{\alpha} \cdots y\left(t_{r}\right)^{\alpha} d t_{1} \cdots d t_{\nu}$ such that $\alpha$ is the highest power occurring in any $\alpha_{\beta}$, and if

$$
\int_{0}^{1} K\left(s, t_{1}, \cdots, t_{\nu}\right) d s \geqq \delta>0 \quad \text { for }\left(t_{1}, \cdots, t_{\nu}\right) \in[0,1]^{\nu} ;
$$

then $\left(^{*}\right)$ has an eigenvalue $\mu_{0}>0$ with eigenfunction $y_{0}(s) \geqq 0$.

Proof. We first state a

Lemma. Consider the mapping $\mathcal{T}:\left(a_{0}, \cdots, a_{n-1}\right) \rightarrow z_{0}$ where $z_{0}$ is the greatest real root of

$$
z^{n}-\sum_{\beta=0}^{n-1} a_{\beta} z^{\beta}=0 .
$$

Then $\varphi$ is defined and continuous on the set $\left\{a_{\beta} \geqq 0 ; 0 \leqq \beta \leqq n-1\right\} \subset E^{n}$.

It is clear that $z_{0}=0$ if and only if $a_{0}=a_{1}=\cdots=a_{n-1}=0$, and $\mathcal{P}$ is continuous at that point. At any other point, however, $z_{0}$ is a simple root which implies continuity of $\mathcal{P}$.

Recalling that $\alpha$ is the highest power of $y$ in any $a_{\beta}$, we observe that $a_{\beta}(s, y)(0 \leqq \beta \leqq n-1)$ exist for all $y(s) \in L_{\alpha}(0,1)$. Moreover, each $a_{\beta}(s, y)$ is a continuous map on $[0,1] \times L_{\alpha}$ into the space $C(0,1)$ of continuous functions on $[0,1]$. For

$$
\left|a_{\beta}(s, y)-a_{\beta}(t, \tilde{y})\right| \leqq\left|a_{\beta}(s, y)-a_{\beta}(s, y)\right|+\left|a_{\beta}(s, \tilde{y})-a_{\beta}(t, \tilde{y})\right|
$$

where the first righthand term can be estimated by expressions of the form

$$
I=\int K\left[y\left(t_{1}\right)-\tilde{y}\left(t_{1}\right)\right]\left(y\left(t_{1}\right)^{\alpha_{1}{ }^{-1}}+\cdots \tilde{y}\left(t_{1}\right)^{\alpha_{1}{ }^{-1}}\right) y\left(t_{2}\right)^{\alpha_{2}} \cdots y\left(t_{\nu}\right)^{\alpha_{\nu}} d t_{1} \cdots d t_{\nu} .
$$

Using Hölder's inequality we arrive at an estimate

$$
|I| \leqq \text { const } \cdot\|y-\tilde{y}\| P(\|y\|,\|\tilde{y}\|)
$$

where $P(u, v)$ is a homogeneous polynomial of order $n-\beta-1$ in $u, v$

8 The treacherous point is that the mapping $\eta \rightarrow y$, [6] p. 252 above, is not continuous. 
and \|\| denotes the norm in $L_{\alpha}$. Thus, $a_{\beta}(s, y)-a_{\beta}(s, \tilde{y}) \rightarrow 0$ uniformly in $s$ as $\tilde{y} \rightarrow y$ in $L_{\alpha}$. The second righthand term in (2) can be estimated by terms

$$
I I=\int\left[K\left(s, t_{1} \cdots t_{\nu}\right)-K\left(t, t_{1} \cdots t_{\nu}\right)\right] \tilde{y}\left(t_{1}\right)^{\alpha_{1}} \cdots \tilde{y}\left(t_{\nu}\right)^{\alpha_{\nu}} d t_{1} \cdots d t_{\nu} ;
$$

so if $|s-t|<\delta(\varepsilon)$, the $K$ 's being continuous, we obtain

$$
|I I| \leqq \varepsilon \int \tilde{y}\left(t_{1}\right)^{\alpha_{1}} d t_{1} \cdots \int \tilde{y}\left(t_{\nu}\right)^{\alpha_{\nu}} d t_{\nu} \leqq \varepsilon\|\tilde{y}\|^{n-\beta}
$$

remembering that $\alpha_{1}+\cdots+\alpha_{2}=n-\beta$ and $\int_{0}^{1} y^{\gamma} d t \leqq\left(\int_{0}^{1} y^{\alpha} d t\right)^{\alpha}$ if $\gamma \leqq \alpha$. This proves continuity of $a_{\beta}$ on $[0,1] \times L_{\alpha}{ }^{9}$. But from the above reasoning it is obvious that $\left\{a_{\beta}(s, y)\right\}$ is an equicontinuous, bounded set of functions if $y$ runs through any bounded set of $L_{\alpha}$. Hence, by the lemma, we have established:

The mapping $y(s) \rightarrow z_{0}(s), z_{0}$ being defined as the greatest real root of (1) for each $s \in[0,1]$, maps any bounded subset of the positive cone in $L_{\alpha}$ onto a set of equicontinuous, non-negative and uniformly bounded functions over $[0,1]$.

Thus the map $y \rightarrow z_{0}$ satisfies the assumptions of Th. 2 if we can show that it is strictly positive. For that end, let $\left\|z_{0}\right\| \rightarrow 0$. If $K$ is the kernel mentioned in our present theorem, we get by (1)

$$
z_{0}(s)^{n} \geqq z_{0}(s)^{\beta} \int_{0}^{1} \cdots \int_{0}^{1} K\left(s, t_{1}, \cdots, t_{\nu}\right) y\left(t_{1}\right)^{\alpha} \cdots y\left(t_{\nu}\right)^{\alpha} d t_{1} \cdots d t_{\nu}
$$

where $\nu \alpha+\beta=n$. Since $z_{0}$ is the greatest real root of (1), there follows

$$
z_{0}(s)^{n-\beta} \geqq \int_{0}^{1} \cdots \int_{0}^{1} K\left(s, t_{1}, \cdots, t_{\nu}\right) y\left(t_{1}\right)^{\alpha} \cdots y\left(t_{\nu}\right)^{\alpha} d t_{1} \cdots d t_{\nu} .
$$

Integrating this last equation, we obtain

$$
\int_{0}^{1} z_{0}(s)^{\nu \alpha} d s \geqq \delta\left[\int_{0}^{1} y(t)^{\alpha} d t\right]^{\nu}=\delta\|y\|^{\nu \alpha} .
$$

Now assume first that $y$ is bounded in $L_{a}$ as $z_{0} \rightarrow 0$. Then $z_{0}$ runs through a uniformly bounded set of continuous functions and hence, as it converges to 0 in measure, $\int_{9}^{1} z_{0}(s)^{\nu \alpha} d s \rightarrow 0$. By (3) this implies $\|y\| \rightarrow 0$. This excludes that $\|y\| \rightarrow \infty$ as $z_{0} \rightarrow 0$, for division of $z$ and $y$ by $\|y\|$ (remember that $\left({ }^{*}\right)$ is homogeneous) would lead to $\|y\|=1$ while $z_{0} \rightarrow 0$.

${ }^{9}$ In general, $\alpha_{\beta}$ are not continuous for the weak topology on $L_{\alpha}$. 
Thus $y \rightarrow z_{0}$ is strictly positive and the application of Th. 2 ends the proof.

3. Let $\Omega$ be a compact region in Euclidean $\mathrm{n}$-space, $C(\Omega)$ the $\mathrm{B}$ space of continuous functions on $\Omega$, and $D(\Omega)$ the space of continuously differentiable functions on $\Omega$ in the topology of uniform convergence of the function and its first order derivatives. Assume a kernel $K(s, t) \geqq 0$ is given on $\Omega \times \Omega$ and a real function $f\left(s ; u, p_{i}\right)$ of $2 n+1$ arguments (letting $\left.s=\left(x_{1}, \cdots, x_{n}\right)\right)$ such that these conditions are satified:

$1^{\circ} . \quad K(\psi)=\int_{\Omega} K(s, t) \psi(t) d t$ is a compact linear transformation on $C(\Omega)$ into $D(\Omega)$ which has an eigenvalue $\lambda_{1}>0$ with an adjoint eigenfunction $\varphi(s)$ that is $\geqq 0$ and bounded except on an $\Omega$-subset of (Lebesgue) measure 0 .

$2^{\circ}$. $f$ is a continuous real valued function, defined for $s \in \Omega, u \geqq 0$, $\left|p_{i}\right|<\infty(i=1, \cdots, n)$ and such that

$$
f\left(s ; u, p_{i}\right) \geqq\left\{\begin{array}{l}
\alpha u \text { if } 0 \leqq u \leqq \delta, \\
K \text { if } \delta<u,
\end{array}\right.
$$

where $\alpha, \delta, K$ are three suitably chosen positive constants. Then the following theorem holds:

Under conditions $1^{\circ}, 2^{\circ}$. the nonlinear integro-differential equation

$$
\lambda u(s)=\int_{\Omega} K(s, t) f\left(t ; u(t), \frac{\partial u}{\partial x_{i}}(t)\right) d t
$$

has for each $c>0$ at least one solution $u \geqq 0$ with $\lambda=\lambda(u)>0$ and $\|u\|=c$, where \|\| denotes a suitably chosen norm of $D(\Omega)$. Moreover, $\lambda(u)$ satisfies the inequality $\lambda \geqq \lambda_{1} \cdot \inf \left(\alpha, K c^{-1}\right)$.

Proof. $D(\Omega)$ is partially ordered by the positive cone $C=\{u: u \geqq$ 0 on $\Omega\}$ but we note that $C$ is not a normal cone (cf. sec. II). For any $\varepsilon>0$, the transformation ${ }^{10}$

$$
T_{\varepsilon}(u)=\int_{\Omega} K(s, t)\left[f\left(t ; u, \frac{\partial u}{\partial x_{i}}\right)+\varepsilon\right] d t
$$

is, due to the continuity of $f$ and condition $1^{\circ}$., compact and continuous on $C$ into $C$. For all $u \in C$ we have

$$
\int_{\Omega} T_{\varepsilon}(u) d s \geqq \iint K(s, t) \varepsilon d t d s \geqq \varepsilon \iint K(s, t) \varphi(s) d t d s=\varepsilon \lambda_{1} \int \rho(s) d s>0
$$

10 The following proof shows how cases may be handled where strict positiveness of the map involved cannot be verified. ( $T_{\varepsilon}$ is not necessarily strictly positive by Example 2 of the preliminary section if $\varepsilon=0$.) 
if $\sup \operatorname{ess} \varphi(s)=1$. Hence $T_{\varepsilon}$ is strictly positive and we may apply Th. 3, hypothesis $\mathrm{H}$ being satisfied through condition $\alpha$. Following the notation of the proof to Th. 3, choose for $q_{1}$ any norm generating the topology of $D(\Omega)$, let $q_{2}=\max |u|$ and write $p=\|\|$. (Obviously $p$ is a norm generating the topology of $D(\Omega)$; it is also evident that, in the present case, the statement of Th. 3 holds for all $c>0$ instead of $0<c \leqq 1$.) Thus, $c$ being fixed, to each $\varepsilon>0$ there is a $u_{\varepsilon} \geqq 0$ and $\lambda_{\varepsilon}>0$ such that

$$
\lambda_{\varepsilon} u_{\varepsilon}=T_{\varepsilon}\left(u_{\varepsilon}\right),\left\|u_{\varepsilon}\right\|=c .
$$

We are going to show that $\lambda_{\varepsilon}$ has a positive lower bound for $\varepsilon>0$. Multiplying (1) by $\varphi$ and integrating, we obtain

$$
\begin{aligned}
\lambda_{\varepsilon} \int_{\Omega} u_{\varepsilon} \varphi d t & =\iint K(s, t) \varphi(s)\left[f\left(u_{\varepsilon}(t)\right)+\varepsilon\right] d s d t \\
& =\lambda_{1} \int \mathcal{P}(t)\left[f\left(u_{\varepsilon}(t)\right)+\varepsilon\right] d t>\lambda_{1} \int \varphi(t) f\left[u_{\varepsilon}(t)\right] d t .
\end{aligned}
$$

Now $\Omega$ is the union of two measurable subsets $\Omega_{1}$ and $\Omega_{2}$ such that $u_{\varepsilon} \leqq \delta$ in $\Omega_{1}$ whereas $u_{\varepsilon}>\delta$ in $\Omega_{2}$. On account of condition $2^{\circ}$. we have

$$
\int_{\Omega_{1}} \varphi f\left[u_{\varepsilon}\right] d t \geqq \alpha \int_{\Omega_{1}} \varphi u_{\S} d t
$$

and

$$
\int_{\Omega_{2}} \varphi f\left[u_{\varepsilon}\right] d t \geqq K \int_{\Omega_{2}} \rho d t \geqq \frac{K}{c \gamma} \int_{\Omega_{2}} \varphi u_{\varepsilon} d t
$$

where $\gamma \leqq 1$ is a constant such that $\max |u| \leqq \gamma\|u\|$ for all $u \in D(\Omega)^{11}$. Hence for the last integral in (2),

$$
\int_{\Omega} \varphi f\left[u_{\varepsilon}\right] d t \geqq \alpha \int_{\Omega_{1}} \varphi u_{\varepsilon} d t+\frac{K}{c \gamma} \int_{\Omega_{2}} \varphi u_{\varepsilon} d t \geqq \inf \left(\alpha_{1} \frac{K}{c \gamma}\right) \int_{\Omega} \varphi u_{\varepsilon} d t
$$

and we finally obtain

$$
\lambda_{\varepsilon} \geqq \inf \left(\alpha, \frac{K}{c \gamma}\right) \cdot \lambda_{1} \quad \text { for } \varepsilon>0
$$

Now let $\varepsilon \rightarrow 0$ in (1). As $\left\|u_{\varepsilon}\right\|=c$ independently of $\varepsilon$, the righthand side of (1) is a relatively compact sequence and so is the corresponding sequence of $u$; by (3). Thus for a common convergent sequence of $\lambda_{\varepsilon}$ and $u_{\varepsilon}$, the limit function $u$ satisfies $\lambda u=T_{0}(u)$ and the proof is complete.

${ }^{11} p=\sup \left\{q_{1}, q_{2},|f|\right\}$ (Th. 3) implies $\gamma \leqq 1$. If $f$ does not depend on any $p_{i}$, the proof may be carried via Th. 2 and we will have $\gamma=1,\|u\|=\max |u|$. 
REMARK. If $f\left(s ; u, p_{i}\right)$ is such that $\delta$ may be chosen arbitrarily large (e.g., if $f=\alpha u+g\left(s ; u, p_{i}\right)$ with $g \geqq 0$ ), then we will have $\Omega_{2}$ void and $\lambda$ will satisfy the inequality

$$
\lambda(u) \geqq \alpha \lambda_{1} .
$$

We apply the preceding theorem to the following problem.

Let $\Omega$ be a compact region in 3 -space such that Green's function for the first boundary problem of potential theory exists. It is then well known that this kernel $G(s, t)$ satisfies condition $1^{\circ}$. of our theorem. It $f\left(s ; u, p_{i}\right)$ is Hölder continuous with respect to all variables, then the equation

$$
\lambda u(s)=\int_{\Omega} G(s, t) f\left(t ; u, \frac{\partial u}{\partial x_{i}}\right) d t
$$

is equivalent to the boundary problem

$$
\Delta u+\lambda^{-1} f\left(s ; u, \frac{\partial u}{\partial x_{i}}\right)=0, u=0 \text { on } \partial \Omega .
$$

Hence, if $f$ satisfies condition $2^{\circ}$., we have:

The nonlinear boundary problem $\left(^{*}\right)$ has, for suitable values $\lambda>0$, solutions $u \geqq 0$ such that $\max u$ attains any positive real number. If, moreover, $f=u+g\left(s ; u, p_{i}\right)(g \geqq 0)$, then for each such $\lambda$

$$
\lambda(u) \geqq \lambda_{1},
$$

where $\lambda_{1}$ is the largest eigenvalue of the corresponding linear problem.

\section{REFERENCES}

1. N. Bourbaki, Élém. de mathém.: Espaces vectoriels topologiques Chap. I, II. Act. Sci. et ind. 1189 (Paris 1953).

2. M. G. Krein and M. A. Rutman, Linear operators leaving invariant a cone in a Banach space (Russian), Uspehi mat. Nauk (N.S.) 3, No. 1 (23), 3-95 (1943). English translation: Amer. Math. Soc. Translat. 26.

3. D. Morgenstern, Thesis (T.U. Berlin 1952, quoted in [6], p. 249).

4. H. Schaefer, Positive Transformationen in halbgeordneten lokalkonvexen Velktorräumen, Math. Annalen 129 (1955), 323-329.

5. —, Halbgeordnete lokalkonvexe Vektorräume, Math. Annalen 135 (1958), 115-141.

6. W. Schmeidler, Algebraische Intgralgleichungen II, Math. Nachr. 10 (1953), 247-255.

7. — Über symmetrische algebraische Integralgleichungen, Annales Acad. Scient. Fennicae, Ser A, I (220) (1956).

8. A. Tychonov, Ein Fixpunltsatz, Math. Annalen 117 (1935), 767-776.

9. E. Rothe, On non-negative functional transformations, Amer. J. Math., 66 (1944), $245-254$ 


\section{PACIFIC JOURNAL OF MATHEMATICS}

\section{EDITORS}

\section{David Gilbarg}

Stanford University Stanford, California

\section{R. A. Beaumont}

University of Washington

Seattle 5, Washington

\section{A. L. Whiteman}

University of Southern California Los Angeles 7, California

L. J. Paige

University of California

Los Angeles 24, California

\section{ASSOCIATE EDITORS}

E. F. BECKENBACH
C. E. BURGESS
E. HEWITT
A. HORN

A. HORN

\author{
V. GANAPATHY IYER \\ R. D. JAMES \\ M. S. KNEBELMAN \\ L. NACHBIN
}

I. NIVEN

T. G. OSTROM

H. L. ROYDEN

M. M. SCHIFFER
E. G. STRAUS

G. SZEKERES

F. WOLF

K. YOSIDA

\section{SUPPORTING INSTITUTIONS}

\author{
UNIVERSITY OF BRITISH COLUMBIA \\ CALIFORNIA INSTITUTE OF TECHNOLOGY \\ UNIVERSITY OF CALIFORNIA \\ MONTANA STATE UNIVERSITY \\ UNIVERSITY OF NEVADA \\ OREGON STATE COLLEGE \\ UNIVERSITY OF OREGON \\ OSAKA UNIVERSITY \\ UNIVERSITY OF SOUTHERN CALIFORNIA
}

\author{
STANFORD UNIVERSITY \\ UNIVERSITY OF TOKYO \\ UNIVERSITY OF UTAH \\ WASHINGTON STATE COLLEGE \\ UNIVERSITY OF WASHINGTON \\ * * * * \\ AMERICAN MATHEMATICAL SOCIETY \\ CALIFORNIA RESEARCH CORPORATION \\ HUGHES AIRCRAFT COMPANY \\ SPACE TECHNOLOGY LABORATORIES
}

Mathematical papers intended for publication in the Pacific Journal of Mathematics should be typewritten (double spaced), and the author should keep a complete copy. Manuscripts may be sent to any one of the four editors. All other communications to the editors should be addressed to the managing editor, L. J. Paige at the University of California, Los Angeles 24, California.

50 reprints per author of each article are furnished free of charge; additional copies may be obtained at cost in multiples of 50 .

The Pacific Journal of Mathematics is published quarterly, in March, June, September, and December. The price per volume (4 numbers) is $\$ 12.00$; single issues, $\$ 3.50$. Back numbers are available. Special price to individual faculty members of supporting institutions and to individual members of the American Mathematical Society: $\$ 4.00$ per volume; single issues, $\$ 1.25$.

Subscriptions, orders for back numbers, and changes of address should be sent to Pacific Journal of Mathematics, 2120 Oxford Street, Berkeley 4, California.

Printed at Kokusai Bunken Insatsusha (International Academic Printing Co., Ltd.), No. 6, 2-chome, Fujimi-cho, Chiyoda-ku, Tokyo, Japan.

PUBLISHED BY PACIFIC IOURNAL OF MATHEMATICS, A NON-PROFIT CORPORATION

The Supporting Institutions listed above contribute to the cost of publication of this Journal, but they are not owners or publishers and have no responsibility for its content or policies. 


\section{Pacific Journal of Mathematics}

\section{Vol. 9, No. $3 \quad$ July, 1959}

Errett Albert Bishop, A minimal boundary for function algebras . . . . . . . . . . . . 629

John W. Brace, The topology of almost uniform convergence . . . . . . . . . . . . 643

Cecil Edmund Burgess, Chainable continua and indecomposability .......... 653

L. Carlitz, Multiplication formulas for products of Bernoulli and Euler

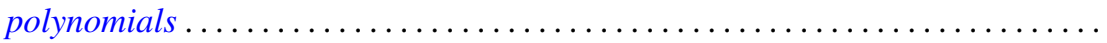

Eckford Cohen, A class of residue systems (mod $r$ ) and related arithmetical

functions. II. Higher dimensional analogues ....................

Shaul Foguel, Boolean algebras of projections of finite multiplicity . . . . . . . . . .

Richard Robinson Goldberg, Averages of Fourier coefficients .................

Seymour Goldberg, Ranges and inverses of perturbed linear operators .

Philip Hartman, On functions representable as a difference of convex functions ....

Milton Vernon Johns, Jr. and Ronald Pyke, On conditional expectation and

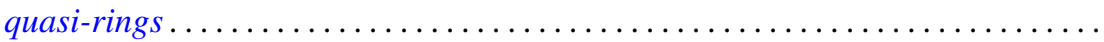

Robert Jacob Koch, Arcs in partially ordered spaces ....................

Gregers Louis Krabbe, A space of multipliers of type $L^{p}(-\infty, \infty) \ldots \ldots \ldots \ldots$

John W. Lamperti and Patrick Colonel Suppes, Chains of infinite order and their

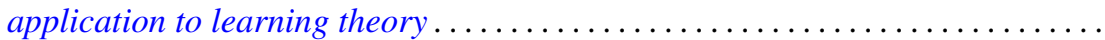

Edith Hirsch Luchins, On radicals and continuity of homomorphisms into Banach

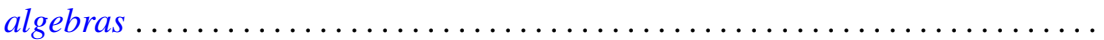

T. M. MacRobert, Multiplication formulae for the E-functions functions of their parameters.

Michael Bahir Maschler, Classes of minimal and representative domains and their kernel functions.

William Schumacher Massey, On the imbeddability of the real projective spaces in Euclidean space.

Thomas Wilson Mullikin, Semi-groups of class $\left(C_{0}\right)$ in $L_{p}$ determined by parabolic differential equations

Steven Orey, Recurrent Markov chains

Ernest Tilden Parker, On quadruply transitive groups ........ . .

Calvin R. Putnam, On Toeplitz matrices, absolute continuity, and unitary

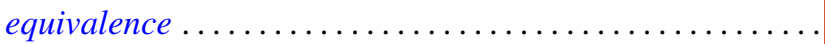

Helmut Heinrich Schaefer, On nonlinear positive operators.

Robert Seall and Marion Wetzel, Some connections between continued fractions and convex sets

Robert Steinberg, Variations on a theme of Chevalley

Olga Taussky and Hans Zassenhaus, On the similarity transformation between a

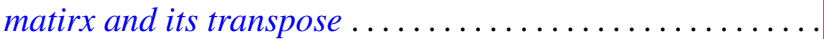

Emery Thomas, The suspension of the generalized Pontrjagin cohomology

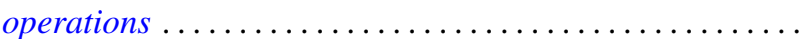

Joseph L. Ullman, On Tchebycheff polynomials ..................... 913

Richard Steven Varga, Orderings of the successive overrelaxation scheme ........ 925

Orlando Eugenio Villamayor, Sr., On weak dimension of algebras . 